\section{Information Technology and Public Health Management of Disasters - A Model for South Asian Countries \\ Dr. Dolly Mathew}

Public School of Social Sciences, Indira Gandhi National Open, Maidan Garhi, New Delhi, INDIA

The paper suggests a model for the developing countries, especially in South Asia, that are faced with common catastrophic events (e.g., floods, earthquakes, epidemics) every year at a large scale causing innumerable deaths, injuries, and damage. The disaster management measures in these countries either are not available or have not been implemented properly. As such, the population remains extremely vulnerable to the adverse health consequences from natural disasters.

Disaster preparedness, emergency relief, and management of disasters are the three main lines of action that provide an effective public-health management of disasters. This could be facilitated by a well-managed global network making use of latest communication technologies that facilitate access to and dissemination of timely information and knowledge for these countries. The model suggested will have its operational utility both at micro and macro levels. At the micro level, the aim will be to utilize modern technology in bringing the internet to the community by setting up of Internet Radio Stations, Community Information Centres, and Community Telecentres. At the macro level, the aim will be to provide health network connectivity between countries of South Asia by stationing a Health Satellite and creation of HDINR (Health and Disaster Information Network) for the Region.

Keywords: Asia; disaster; disaster management; global network; information; internet; natural disasters; public health; technology

E-mail: dollymathew@hotmail.com
Tele-consultation at Emergency Department:

Telemedicine Experience in Taiwan

Lee-Min Wang, MD; ${ }^{1}$ Hong-Chang Lo, MD; ${ }^{1}$ ChenHsen Lee, MD; ; Jen-Hua Hung;, Dab-Dian Tang ${ }^{2}$

1) Emergency Department; 2) Information Services Center, Veterans General Hospital-Taipei, National Defense Medical Center.National Yang-Ming University, Taipei, Taiwan, ROC

Objective: To evaluate the use of telemedicine in the emergency departments between Taipei-Veterans General Hospital and Kinmen-Granite County Hospitals in the Taiwan area.

Methods: Patients were chosen from 1997 to 1999. A total of 231 tele-consultation cases were collected. The evaluation criteria were: 1) the quality of the consultation; 2) the duration of the tele-consultation; 3) the time required of preparation for the available and transferring materials; 4) the status of the available equipment; 5) the availability of the computer function; 6) the lack of the clear materials; 7) the satisfaction of the doctors; 8) the benefit to the patients; and 9) the charges.

The purposes of the project were to establish computer networks between selected hospitals and through the high speed communication and high power computer processing, to electronically exchange medical information, and to conduct clinical examination and consultation. It aimed to provide on-time, quality medical services to patients in the remote rural areas such as villages and small towns in the mountains, and on the coasts and islets distant from Taiwan. It also was intended to facilitate onthe-job training and education for doctors in those areas. Results: We collected the cases of the tele-consultation from the available telemedicine between Taipei VGH and Kinmen-Granite County Hospital from July 1997 to June 1999. The data included 253 Taipei-VGH doctors and 253 Kinmen doctors. The peak age of the participants was 21-30 years. The most involved medical section was radiology, followed by chest medicine, dermatology, orthopedics, neurosurgery, general surgery, etc. The questionnaire results: 1) Searching for a second opinion, $72.3 \%$; 2) The compliance of the patients after receiving the teleconsultation, $>89 \%$; 3) $>97 \%$ of the participating doctors think telemedicine system will affect the quality of the consultation and that it is helpful. The doctors in Kinmen Hospitals were more satisfied with the teleconsultation facility than were the doctors at the Taipei-VGH.

Conclusions: From the questionnaire, the consultation doctors confirm that the clinical evaluation of the telemedicine is positive. It is intended to facilitate on-thejob training and education for the doctors in the teleemergency medicine. The patients and their family believe that telemedicine is helpful, and free of charge after the teleconsultation in remote areas.

Keywords: consultation; emergency medicine; questionnaire; telecommunication; telemedicine 